\title{
Culture, Food Communication and School Nutrition Policies: A portfolio for Improving Youth Dietary Patterns - Case of Beirut City
}

\author{
S. Hamadeh ${ }^{1}$ and M. Marquis ${ }^{2}$ \\ ${ }^{1}$ Haigazian University, Mexique Street, Beirut, Republic of Lebanon and \\ ${ }^{2}$ Montreal University, Côte-Sainte Catherine street, Montreal, Canada
}

Culture and society are essential in shaping a person's lifestyle ${ }^{(1)}$. Precisely, food communication can influence, positively or negatively, youth' food choices and patterns ${ }^{(2)}$. Schools have proved a valuable setting in which to promote nutrition and health amongst youth ${ }^{(3)}$. The primary aim of this study was to qualitatively explore the perceptions of multidisciplinary Lebanese key stakeholders on nutrition communication and school nutrition policies (SNP) impact on youth lifestyles in metropolitan contexts.

In order to explore these perceptions, directed and semi-structures individual interviews with key stakeholders $(\mathrm{n}=64)$ in conjunction with focus groups $(\mathrm{n}=2)$ with parents and teachers from 2 schools $(1$ private and 1 public) in Beirut city were employed. Collected data have been submitted to a thematic qualitative analysis.

All participants perceived the transformation of the global communications and information landscape raises new challenges including extensive food marketing to youth. This study helps local and national efforts: 1) to improve the healthfulness of food choices in a variety of spots and points of sales in cities and, 2) to inform regulatory options and, food communication and SNP implications. The table outlines major participants' suggestions for improvement of youth dietary patterns in metropolitan contexts.

Participants' suggestions for improving youth lifestyles in metropolitan contexts

Identify and analyze sociocultural, environmental, behavioral, economic and political determinants of youth lifestyles.

Identify appropriate areas for action in different sociocultural contexts.

Work with the multiple role models, decision makers and nutrition gatekeepers influencing youth lifestyles.

Build workforce capacity for effective SNP deployment and evaluation.

Assess of the internal and external school environments.

Deploy a strategy to layer and repeat nutrition messages through multiple channels (e.g. visual marketing materials linked to in-class nutrition education program).

Need for more stringent government regulations on food communication and marketing "directed to youth" (e.g. prohibition of direct corporate ads on school space).

Such perceptions offer the potential for future enhancement of the effective food communication strategies and SNP, and improvements in their efficacy to be made. Sociocultural-based nutrition communication strategies and SNP that pay attention to specific health behavioral goals, target populations, communication activities and innovative techniques for feedback and evaluation can help to improve youth dietary patterns and prevent diseases in adulthood.

1. Wennberg M, Gustafsson P, Wennberg P et al. (2014) Public Health Nutr 18(1): 122-129.

2. Freeman B, Kelly B, Vandevijvere S et al. (2016) Health Prom Intl 31, 954-961.

3. Hamadeh S \& Marquis M (2017) Nutr Health Sc 4(1): 9p. 